\title{
Comparison of Several Global Mixing Performance Metrics for High-Speed Fuel Injectors
}

\author{
Cody R. Ground*, Tomasz G. Drozda ${ }^{\dagger}$, Karen F. Cabell ${ }^{\ddagger}$, Erik L. Axdahl ${ }^{\ddagger}$ \\ NASA Langley Research Center, Hampton, VA, 23681
}

To experimentally assess and compare the mixing performance of high-speed fuel injectors for scramjet engines, quantitative global metrics are needed. The one-dimensional metric most commonly used to assess the degree of mixing completeness at a given downstream station is the mixing efficiency parameter. The experimental determination of the mixing efficiency parameter requires measurement of the spatial distributions of both the fuel mass fraction and the mass flux. Standard in-stream gas sampling techniques can be used to measure the fuel mass fraction distribution, however the mass flux distribution is not easily determined experimentally because it requires the measurement of three independent aerothermodynamic variables in addition to the mixture composition. For this reason, several metrics that can be calculated from the fuel distribution alone are commonly used to assess mixing performance. Because these other metrics do not provide a mass flux-weighted measure of the local degree of mixing completeness, they may not correlate well with the mixing efficiency parameter. Therefore, if the substitute metrics are to be used to compare the mixing performance of candidate fuel injector concepts, it is important to understand their relationships to the mixing efficiency parameter in a representative scramjet combustor flowfield. This work investigates the relationships between the mixing efficiency parameter and several substitute metrics that are able to be measured with the current experimental setup of the Enhanced Injection and Mixing Project at the NASA Langley Research Center for baseline strut and ramp injectors. The results of these comparisons have revealed that it is possible to glean different (i.e., incorrect) conclusions about which injector is the better mixer when the substitute mixing performance metrics are used instead of the mixing efficiency parameter, thereby highlighting the importance of mass flux-weighted mixing performance metrics.

\section{Nomenclature}

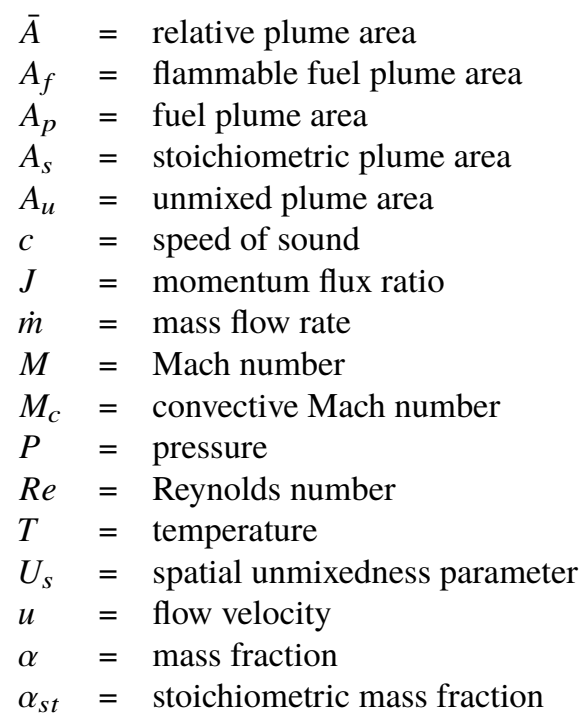

\footnotetext{
*Research Aerospace Engineer, Hypersonic Airbreathing Propulsion Branch, Member AIAA.

${ }^{\dagger}$ Research Aerospace Engineer, Hypersonic Airbreathing Propulsion Branch, Associate Fellow AIAA.

${ }^{\ddagger}$ Research Aerospace Engineer, Hypersonic Airbreathing Propulsion Branch, Senior Member AIAA.
} 


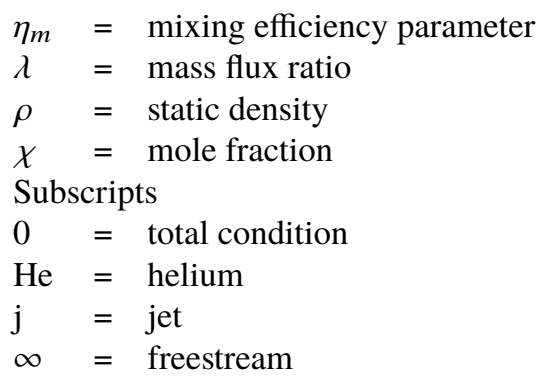

\section{Introduction}

$\mathrm{T}$ HE feasibility of achieving sustained scramjet-powered flight has been proven by the successful flight demonstrations of the X-43 (Mach 7 and 10) and X-51 (Mach 5) vehicles [1, 2]. However, in order to move beyond technology demonstration and make scramjet-powered flight into a normative operation, several technical challenges must be addressed. A primary challenge is reducing the combustor length in order to reduce the vehicle weight and thermal load. This can be accomplished by the development of fuel/air mixing enhancement strategies. Enhancing the rate at which fuel and air mix is of paramount importance because it is the rate-limiting step of the combustion process in a scramjet engine [3]. To this end, past research efforts have devised and studied various fuel/air mixing enhancement strategies and injector configurations in supersonic flows. Many of these strategies have been reviewed previously by Seiner et al. [4], Gutmark et al. [5], and Lee et al. [6]; however, a deeper understanding of the physics driving the fuel/air mixing process in the scramjet combustor is required in order to design fuel injection systems that sustain and enhance engine performance across a wide range of flight Mach numbers at both on-design and off-design conditions.

The Enhanced Injection and Mixing Project (EIMP) [7], currently underway at the NASA Langley Research Center, is a study on the underlying fundamental physics of fuel injection and mixing relevant to scramjets with flight Mach numbers greater than 8 . An ultimate objective of the project is to develop mixing enhancement strategies that improve injector performance by increasing mixing efficiency while minimizing the drag and total pressure loss incurred during the fuel/air mixing process. A highly coupled experimental and computational approach is used in the EIMP. The experiments, which are performed in the Arc-Heated Scramjet Test Facility (AHSTF) at NASA Langley, are intended to screen fuel injector concepts and to anchor computational fluid dynamics (CFD) simulations. Measurements include in-stream gas sampling, pitot pressure, and total temperature measurements as well as flow visualization using the nitric oxide planar laser-induced fluorescence (NO PLIF) technique. The CFD simulations of the mixing flowfields (under both experimental and notional flight conditions) provide detailed flowfield information which cannot be measured in the experiments and allow for the calculation of performance metrics. Thus far, three different types of fuel injectors, a strut, ramp, and flushwall injector, have been investigated both experimentally [8, 9] and computationally [10-12]. These injectors were chosen as baselines for an initial study and represent the three basic classes of injectors commonly considered for high speed propulsive flowpaths.

To fairly compare the performance of different injector configurations, a global metric that assesses the local degree of mixing completeness at a given downstream station is needed. By basing comparisons on a global metric that is one-dimensional, varying only in the primary flow direction, it is possible to determine which injector configuration more rapidly mixes the injected fuel and air. The quantitative metric that is best suited for this non-reacting application is the mixing efficiency parameter (henceforth referred to simply as "mixing efficiency," for brevity). This mass flux-weighted parameter is defined as the fraction of least available reactant that would react if the fuel/air mixture were brought to chemical equilibrium without further local or global mixing. Although the fuel distribution is relatively easy to measure experimentally, the mass flux distribution is not. Its calculation requires the measurement/knowledge of the spatial distribution of three independent aerothermodynamic properties in addition to gas composition. For this reason, several substitute metrics that can be determined from fuel distribution alone are commonly used as indicators of mixing performance in lieu of mixing efficiency. However, because these other metrics do not provide a mass flux-weighted measure of mixing completeness, they could lead to different conclusions from those based on mixing efficiency. Therefore, it is important to understand their relationship to mixing efficiency if the substitute metrics are to be used to draw conclusions about the mixing performance of various fuel injector concepts.

The mass flux distribution, and therefore mixing efficiency, cannot be determined from the planned measurements in the EIMP experiments. However, it is desirable to use the experimentally measured fuel distribution to be able to compare and rank the relative performance of various injector concepts. Therefore, this work investigates the relationship 
between mixing efficiency and several substitute metrics that are able to be calculated from only the spatial helium mass fraction distribution. Ideally, one (or multiple) of these substitute mixing performance metrics will have a strong linear correlation with the mixing efficiency for each injector configuration while also maintaining similar relative magnitudes for each configuration. This would allow for the same conclusions that would be drawn by comparing injectors using mixing efficiency to be made with a substitute metric that is more readily measured. The metrics that will be compared to mixing efficiency are the spatial unmixedness parameter, decay of maximum fuel mass fraction, and three plume area-based metrics. These metrics have been utilized in previous fuel/air mixing studies in the literature for a wide variety of injector types, including pylon, ramp, and aeroramp configurations [13-15]. However, the degree to which the various metrics correlate with the mixing efficiency, when comparing different injector configurations, was not detailed in these works.

The comparisons between mixing efficiency and the substitute metrics will be made using the results of CFD simulations previously performed for the strut and ramp fuel injectors at the experimental test conditions. The mixing efficiency and substitute metrics will be calculated by extracting data from the CFD solutions at the locations which will be probed experimentally so that a synthetic "experimental" data set is obtained. In the planned experiments, the gas sample probes will be spaced at $1 / 8$ inch in the vertical and horizontal directions across selected planes downstream of the injector. In the present analysis, several synthetic experimental data sets will be extracted with different sample grid spacings in order to assess the effect that the spatial resolution of the gas sample measurements has on the values of the one-dimensional metrics. The synthetic experimental data will be used to calculate the substitute metrics as if they were actual experimental data. These metrics will be compared to the mixing efficiency that has been calculated from the full resolution CFD solution in order to determine which of these metrics have the highest correlation with the mixing efficiency.

The results of this study are expected to serve as a guide for the measurement (e.g., determining the measurement spacing required for an acceptably accurate calculation of the various substitute metrics) and interpretation (e.g., determining which substitute metric best correlates with mixing efficiency) of these metrics in future experimental campaigns. This is important because future efforts of the EIMP might rely solely on the experimental determination of these metrics to screen various injector configurations.

\section{Experimental Facility, Measurement Hardware, and Injector Configurations}

The EIMP experiments utilize a nominally Mach 6 nozzle to simulate the aerodynamic Mach number at the entrance of a scramjet combustor. The actual nozzle exit Mach number $(\approx 6.4)$ and Reynolds number are consistent with the combustor entrance conditions for a notional vehicle at a flight Mach number of 15 and a dynamic pressure of 1500 psf [12]. To reduce experiment cost and complexity, the tests are conducted at a reduced total temperature with an inert fuel simulant (helium) to allow for uncooled test hardware. This approach has long been used in non-reacting flows to analogously study the fundamental mixing processes that take place in a scramjet combustor in the absence of heat release [14-16]. Though the total temperature of the ground test condition has been dramatically lowered (approximately, by a factor of 9 with respect to the simulated flight Mach number), it is important to note that the Reynolds numbers between the ground experiments and the notional flight condition are comparable, within $15 \%$. Additionally, Drozda et al. [11] have quantified the effect that reducing the total enthalpy has on the mixing behavior for these specific injector geometries and flow conditions via a set of CFD simulations. These simulations found that the "cold-flow" experiments acceptably replicate the global mixing behavior of the simulated notional flight condition.

The injector configurations are tested on an open flat plate to allow for easy optical and in-stream probe access to the fuel/air mixing region. The effects of testing these configurations on an open flat plate instead of in a closed ducted flowpath have been computationally investigated by Drozda et al. [12]. A schematic of the experimental apparatus is shown in Fig. 1. The flow is from left to right. The test bed flat plate features a rectangular opening for mounting interchangeable injector blocks. The injector blocks, in general, can accommodate a spanwise row of several injectors. The facility air flows over the injector bodies and mixes with helium downstream of the injection plane. Also depicted in Fig. 1 are the jet stretchers which are intended to extend the downstream extent of the undisturbed nozzle exit flow to prevent over- and underexpansion of the facility air flow upstream of the instrumentation survey plane.

The gas sampling, pitot pressure, and total temperature data will be obtained using in-stream probes mounted on an automated rake traverse system that translates across the mixing flowfield at selected $y z$ planes perpendicular to the free stream flow direction. A local gas sampling and analysis system (GSAS) will provide the helium mass fraction of the gas captured by forty gas sample probes at each rake position. The end result of the experimental measurements will be two-dimensional contour maps of the helium mass fraction, pitot pressure, and total temperature distributions at multiple 
planes downstream of each injector configuration tested. These data will not only allow for a quantitative comparison of the multiple injector configurations tested, but will also allow for quantitative comparisons of the experimental results and CFD simulations to be made. The in-stream measurements can be obtained within a 20 inch long measurement volume adjacent to the test bed plate within a $5 \times 5 \mathrm{in}^{2}$ cross section. The rake traverse system is depicted in its farthest downstream location in Fig. 1. Fig. 22 shows a more detailed view of the two gas sample rakes (which double as the pitot probe rakes) and the total temperature rake. Each gas sample rake, A and B, contains 20 probes spaced at $1 / 4$ inch in the vertical $y$ direction. The Rake A probes are offset by $1 / 8$ inch from the Rake B probes so that, when the measurements from both rakes are combined, the final result is a grid of 40 measurements that are vertically spaced at $1 / 8$ inch with the first measurement being 0.4 inch from the wall. The interested reader is referred to the work of Cabell et al. [7] for a more detailed description of the facility, test conditions, and instrumentation hardware.

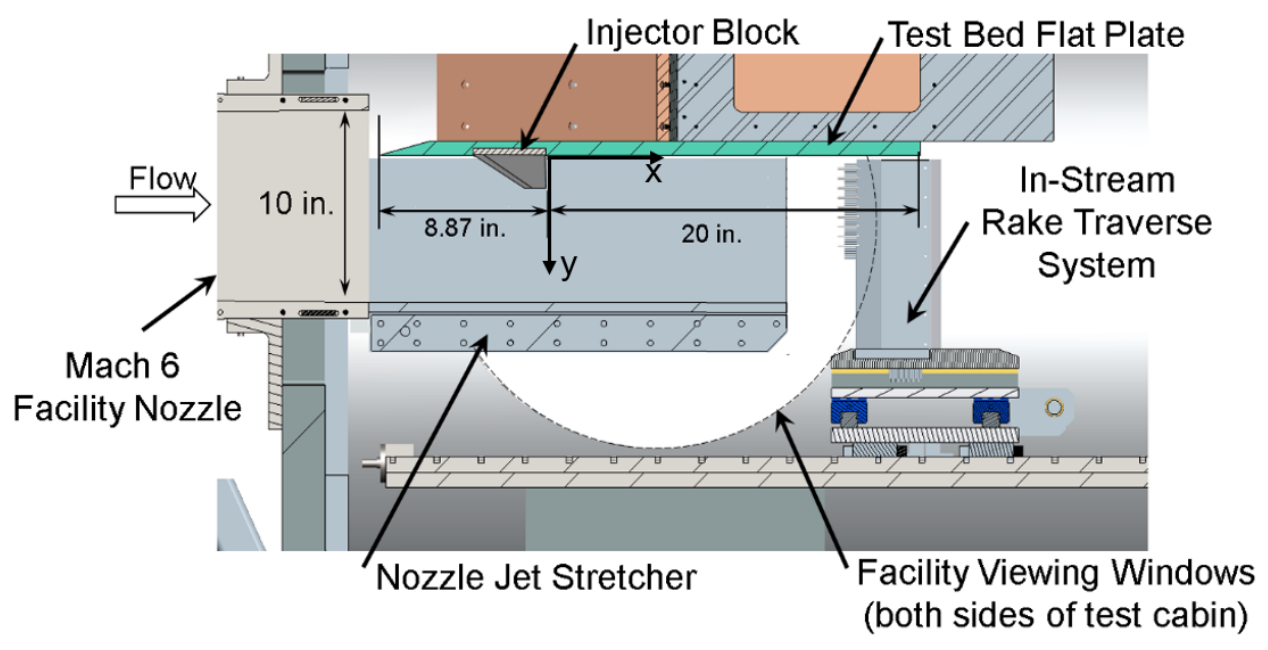

Fig. 1 Schematic of the test cabin showing the facility nozzle, flat plate, injector block, jet-stretchers, and the in-stream rake system. The facility viewing window is denoted by the dashed line.
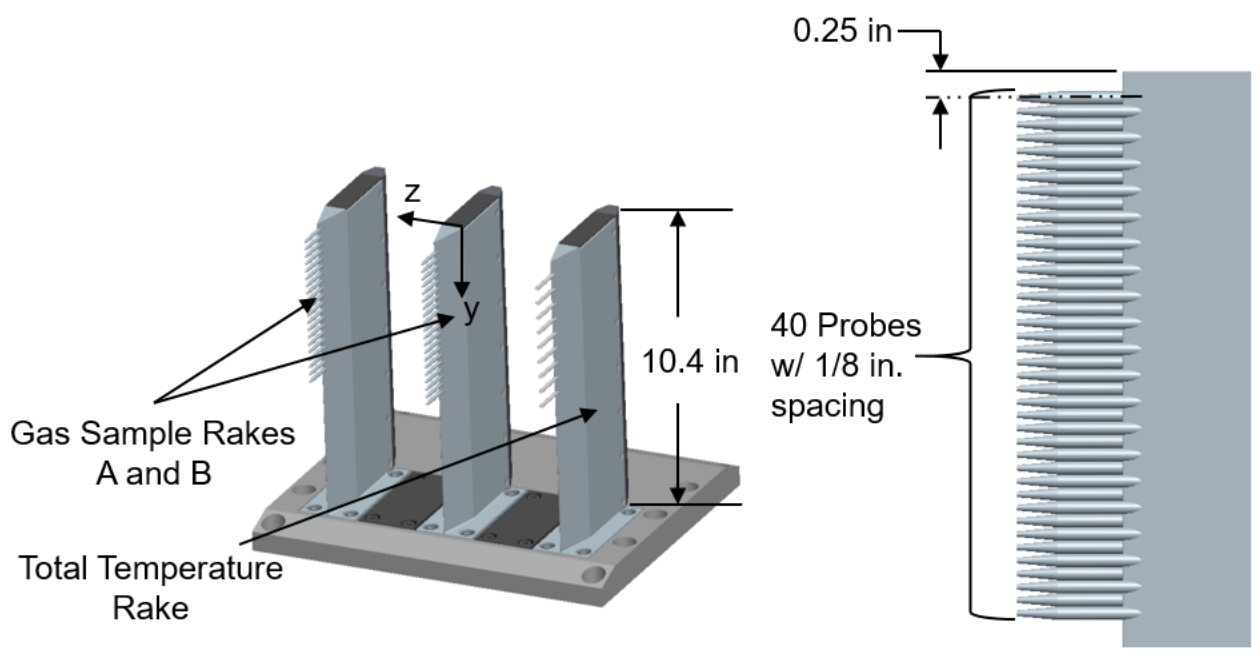

Fig. 2 In-stream measurement rakes schematic.

The analysis presented herein is limited to the EIMP baseline strut and ramp injectors. These injectors use different strategies to distribute and mix the injected helium into the supersonic crossflow. Strut-type injectors generally provide 
two distinct benefits in hypervelocity applications. First, because they penetrate into the supersonic crossflow of the combustor, they place fuel directly into the core air flow. Second, the fuel is generally injected parallel to the primary flow direction, thereby augmenting the thrust of the engine. The primary drawbacks of the strut-type injector are the additional drag and total pressure loss generated by the intrusive nature of the injector body and the high heat loads on the surfaces of the injector. A three-view drawing of the baseline strut injector is shown in Fig. 3 . Each of the four circular injector ports on the aft face of the strut injector are designed to expand helium to Mach 3 and have a throat diameter of 0.083 inch, followed by a conical expansion with a $6^{\circ}$ half-angle. The top injection port in the strut injects fuel at an angle of $20^{\circ}$ relative to the freestream, while the others are parallel to the freestream.

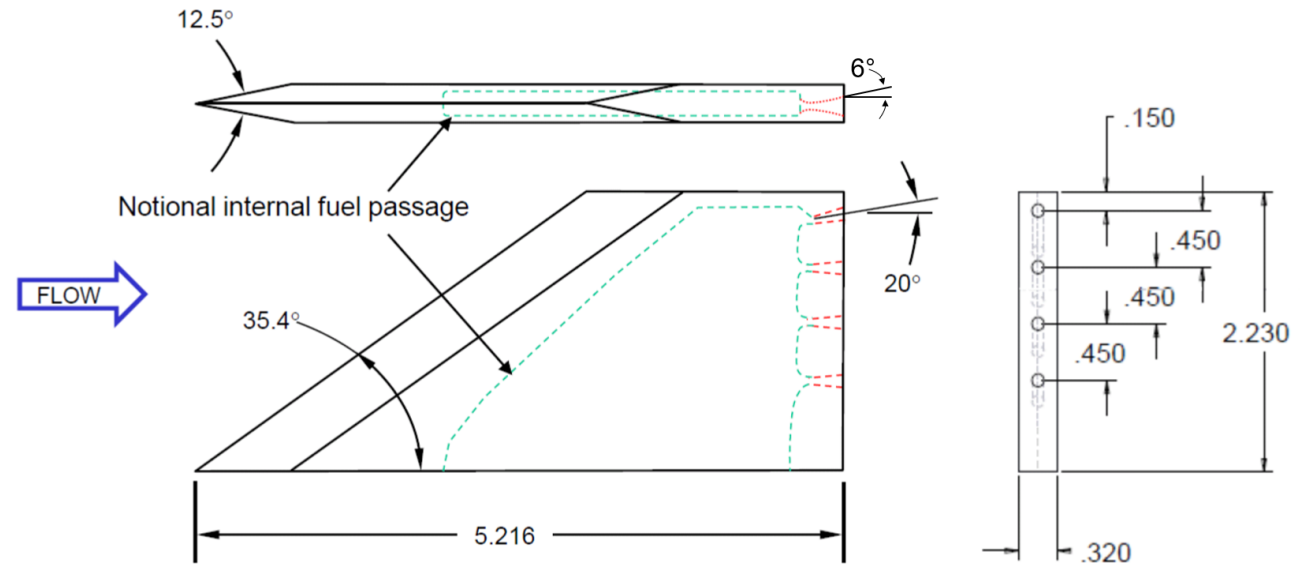

Fig. 3 Three view drawing of baseline strut injector; dimensions in inches.

The second injector configuration is an unswept ramp injector. Compared to the strut injector, the ramp injector does not protrude as far into the flow. The ramp-shaped body of the injector is designed to produce a counter-rotating vortex pair (CVP). The CVP is formed as the flow spills from the higher pressure upper surface of the ramp to the lower pressure flow on either side of the ramp. This process results in the roll-up of the vortex pair that convects the fuel, which is usually injected parallel to the flow at the base of the ramp, upward toward the center of the combustor. This process also stretches and deforms the injected plume, thereby increasing the interfacial surface area available for the fuel and air to mix. A three-view drawing of the baseline ramp injector is shown in Fig. 4 . Four circular injector ports, which are angled upward and to the side (by $11.8^{\circ}$ and $10^{\circ}$, respectively) in order to promote injection of fuel toward the CVP, are located on the aft face of each ramp. Each injector port has a throat diameter of 0.108 inch followed by a conical expansion with a half-angle of $10^{\circ}$ that is designed to expand helium to Mach 3 . A summary of the freestream air and fuel injection conditions used in the CFD simulations is given in Table 1.

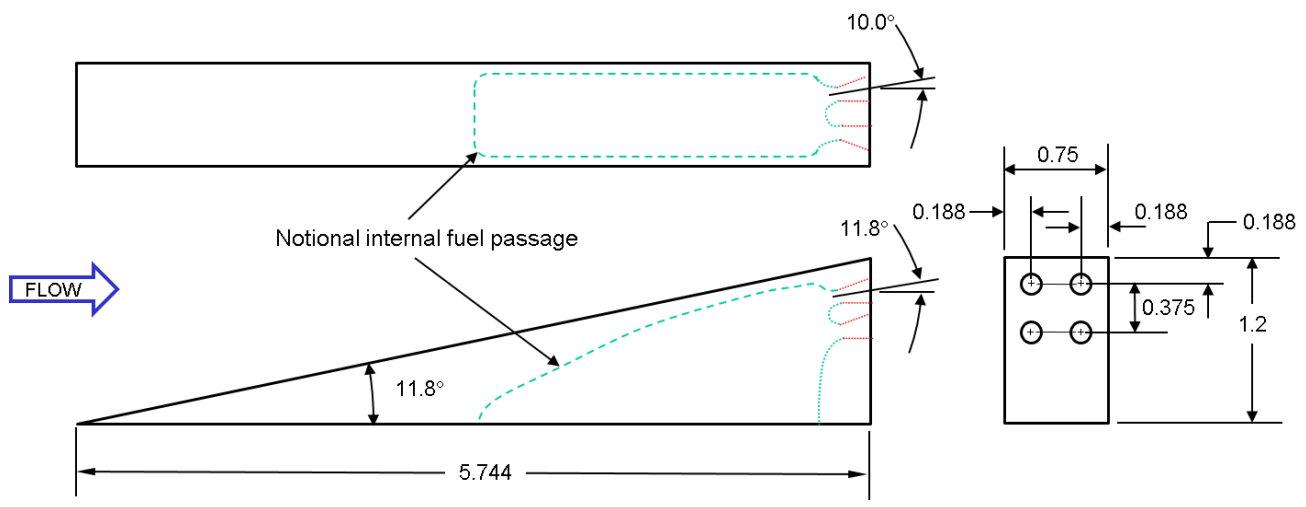

Fig. 4 Three view drawing of baseline ramp injector; dimensions in inches. 
Table 1 Nominal global parameters of interest for each injector configuration

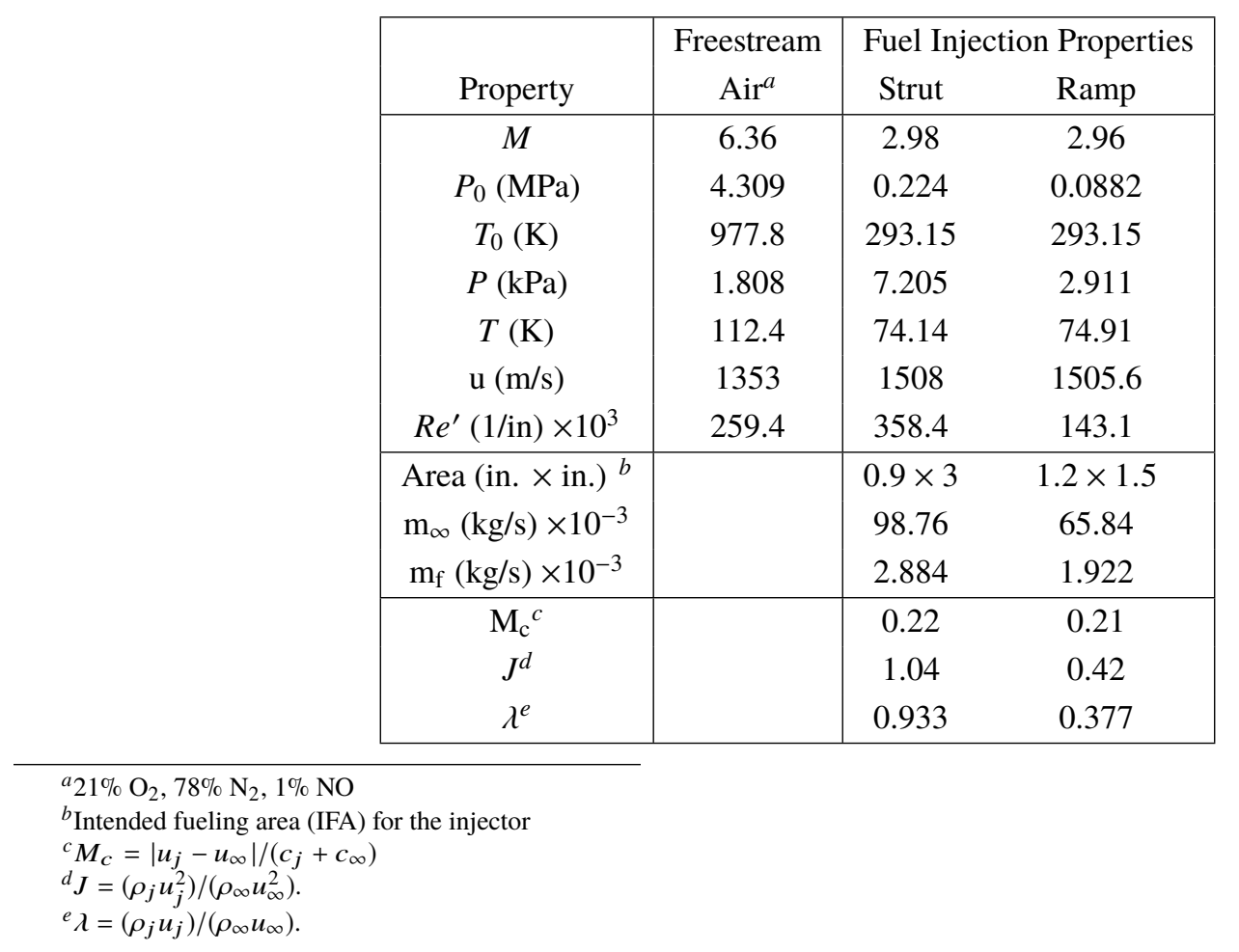

\section{Computational Simulations}

Results of the previously performed CFD simulations of the strut and ramp injector are provided by Drozda et al. [10-12], however important modeling details are repeated here and selected results are shown for illustrative purposes. Reynolds-averaged simulations were performed using the Viscous Upwind aLgorithm for Complex flow ANalysis (VULCAN-CFD) code [17]. VULCAN-CFD is a multiblock, structured-grid, cell-centered, finite-volume solver widely used for all speed flow simulations. The advective terms were computed using a Monotonic Upstream-Centered Scheme for Conservation Laws (MUSCL) scheme [18] with the Low-Dissipation Flux-Split Scheme (LDFSS) of Edwards [19]. The thermodynamic properties of the mixture components were computed using curve fits of McBride et al. [20]. The governing equations were integrated using an implicit diagonalized approximate factorization (DAF) method [21]. The baseline blended $\mathrm{k}-\omega / \mathrm{k}-\epsilon$ turbulent physics model of Menter [22] was used. The Reynolds heat and species mass fluxes were modeled using a gradient diffusion model with turbulent Prandtl and Schmidt numbers of 0.9 and 0.5, respectively. These values were set based on experience and best practice with similar flows. Wilcox wall matching functions [23] were also used, however, their implementation in VULCAN-CFD includes a modification that allows the simulations to recover the integrate-to-the-wall behavior as the value of normalized wall-distance, $y+$, approaches one. All simulations were converged until the total integrated mass flow rate and the total integrated heat flux on the walls remained constant to within six significant digits. This typically occurred when the value of the $\mathrm{L}_{2}$-norm of the steady-state equation-set residual decreased by about 4-5 orders of magnitude. Grid dependence analyses for the numerical simulations of both the strut and ramp injectors were previously assessed by Drozda et al. [10]. The numerical simulations took advantage of the geometric symmetries of the injectors, therefore, only a single injector is simulated with the symmetry boundary conditions used at the midplane between the adjacent injectors.

Mach number contour plots in the center $x y$ plane of each injector are shown in Fig. 5 These contour plots illustrate the dominant flow features generated by each injector's interaction with the Mach 6 freestream flow. Fig. 6 shows cross sectional views of the helium mass fraction distribution at different $y z$ planes downstream of each injector. The extent of the plotted domains represent the intended fueling area for each injector. The contours of $\alpha_{H e}$ are cut off below a helium mole fraction of $\chi_{\mathrm{He}}=0.01$ ( $\alpha_{\mathrm{He}} \approx 0.0014$ ), which is the expected minimum sensitivity of the GSAS measurements. The black isocontour lines in both Figs. 5 and 6 denote a helium mass fraction equal to the stoichiometric value for hydrogen $\left(\alpha_{s t}=0.0285\right)$. 

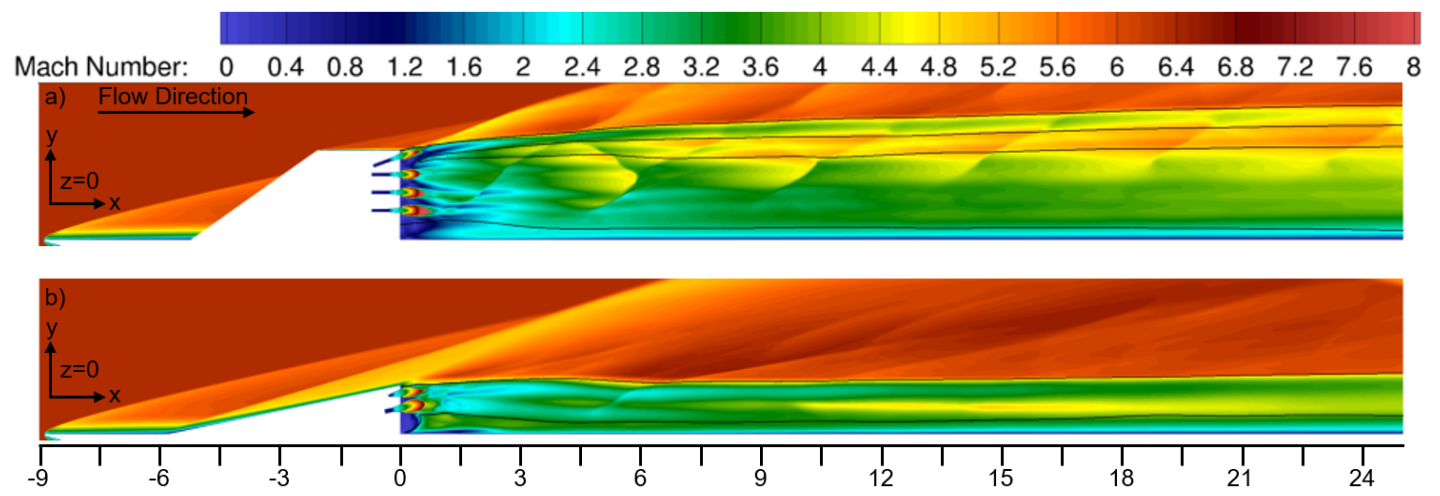

Fig. 5 Mach number contours for center $x y$ plane $(z=0)$ for baseline a) strut injector and b) ramp injector, dimensions in inches.
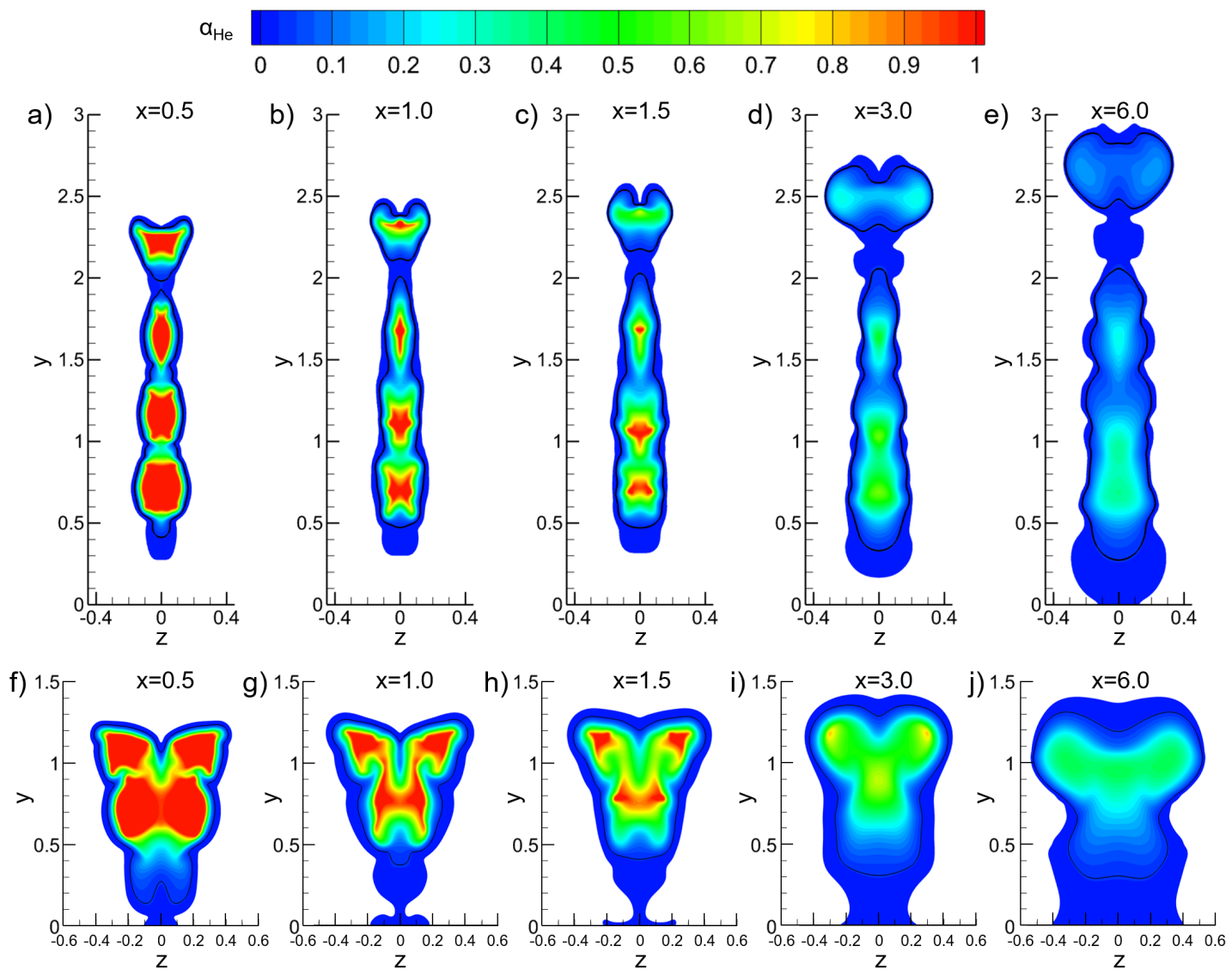

Fig. 6 Helium mass fraction contours for $y z$ planes at different downstream distances for baseline a)-e) strut injector and f)-j) ramp injector, dimensions in inches.

For the strut injector, the Mach number contours show that the injected helium interacts with a complex system of reflecting shock and expansion waves. These waves are generated by the interaction of the strut's leading edge oblique shock wave with both the body of, and the shock wave generated by, the adjacent fuel injector. The helium mass fraction 
contours for the strut show that the fuel plume rapidly expands and the injected helium diffuses throughout the intended fueling area as the flow progresses downstream. The bottom two jets of helium interact and begin to coalesce by the $x=3.0$ inch downstream station. An interesting feature is evident in both the Mach number and helium mass fraction contours at the upper edge of the strut injector. They show that the uppermost helium jet is entrained upward into the core flow of freestream air by the CVP shed by the strut's upper surface. This further increases the penetration of the fuel injected by the strut and leads to more rapid mixing of the helium injected through this specific port.

For the ramp injector, both the Mach number and helium mass fraction contours show that the upward penetration of the fuel plume is limited. This is due to the fact that the CVP generated by the ramp is constrained by the narrow spacing between the fuel injectors; nevertheless, the fuel plume does expand and diffuse rapidly in the $z$ direction, nearly filling the entire intended fueling area. Though its overall upward penetration is limited, the helium mass fraction contours do show that the CVP entrains the helium injected through the bottom two fuel ports upwards towards the top of the fuel plume. For further details on the CFD simulations and discussion of their results, the reader is referred to the previous works of Drozda et al. [10,12].

\section{Global Mixing Performance Metrics}

The global mixing performance metrics that will be compared in this work are defined in this section. The substitute metrics that will be compared to the mixing efficiency are the spatial unmixedness parameter, decay of maximum fuel mass fraction, and three plume area-based metrics. Estimations of the continuum values of these metrics have been made using the Richardson extrapolation technique [24] after extracting data from the converged CFD solution on uniform grids with coarse $\left(1 / 16 \times 1 / 16\right.$ in. $\left.^{2}\right)$, medium $\left(1 / 32 \times 1 / 32\right.$ in. $\left.^{2}\right)$, and fine $\left(1 / 64 \times 1 / 64\right.$ in. $\left.{ }^{2}\right)$ spacings. Plots of the Richardson extrapolated values of all metrics versus downstream distance for both the strut and ramp injector configurations can be found at the end of this section in Fig. 7 .

\section{A. Mixing Efficiency Parameter}

The mixing efficiency, $\eta_{m}$, is defined as the fraction of least available reactant (i.e., either $\mathrm{O}_{2}$ or fuel) that would react if the fuel/air mixture were brought to chemical equilibrium without further local or global mixing. Thus, mixing efficiency requires a two-part definition dependent upon whether the flow is globally fuel-lean or fuel-rich. The definition used herein is the one proposed by Mao et al. [25], i.e.,

$$
\eta_{m}=\frac{\int \alpha_{R} \rho u d A}{\int \alpha \rho u d A}
$$

where,

$$
\alpha_{R}= \begin{cases}\alpha, & \alpha \leq \alpha_{s t} \\ \frac{\alpha_{s t}}{1-\alpha_{s t}}(1-\alpha), & \alpha>\alpha_{s t}\end{cases}
$$

In the formulation above, the integration is over a single downstream $y z$ plane and $\alpha$ is either the fuel or oxidizer mass fraction depending on whether the mixture is globally fuel-lean or fuel-rich, respectively. Because the experiments are performed on an open plate instead of a closed duct, an intended fueling area (IFA) for each injector configuration must be defined in order to define the global equivalence ratio. The definition of the IFA is discussed at length in Drozda et al. [12]. In the current work, the global equivalence ratio over the intended fueling area is equal to 0.75 for each injector configuration. Therefore, in Eq. 1 and Eq. 2, $\alpha$ denotes the mass fraction of helium and $\alpha_{s t}$ is taken to be 0.0285 , which is the stoichiometric hydrogen mass fraction for hydrogen/air mixtures. The mixing efficiency varies from 0 to 1 with $\eta_{m}=0$ corresponding to a perfectly segregated mixture and $\eta_{m}=1$ corresponding to a perfectly mixed mixture.

Fig 7 a depicts the Richardson extrapolated mixing efficiency for the two injector configurations. These mixing curves show that the strut injector mixes the fuel and air in the near field ( $x<3$ inch) much more rapidly than the ramp injector. Beginning around $x=7$ inch, and proceeding for the remainder of the downstream distance simulated, the ramp and strut injectors mix the fuel and air at approximately the same rate, meaning that the majority of the mixing enhancement achieved by the strut injector occurs in the near field in the strut's wake. 


\section{B. Spatial Unmixedness Parameter}

The first of the substitute metrics that will be compared to the mixing efficiency is the spatial unmixedness parameter, $U_{s}$. This parameter was defined by Liscinsky et al. [13] with the purpose of serving as a global mixing performance metric for planar laser-based fuel plume imaging experiments where only fuel concentration measurements were obtainable. Here the form proposed by Fuller et al. [14] will be used:

$$
U_{s}=\frac{\alpha_{v a r}}{\alpha_{e q}\left(1-\alpha_{e q}\right)},
$$

where,

$$
\begin{gathered}
\alpha_{v a r}=\frac{1}{n} \sum_{i=1}^{n}\left(\bar{\alpha}_{i}-\alpha_{e q}\right)^{2}, \\
\alpha_{e q}=\frac{\dot{m}_{j}}{\dot{m}_{j}+\dot{m}_{\infty}}, \\
\bar{\alpha}_{i}=\alpha_{i} \alpha_{e q}\left(\frac{1}{n} \sum_{i=1}^{n} \alpha_{i}\right)^{-1} .
\end{gathered}
$$

From inspection of Eq. 3 , it is seen that the spatial unmixedness parameter is simply a ratio of the spatial variance in fuel mass fraction at a given flow plane to the maximum spatial variance of the same quantity. Therefore, $U_{s}=0$ corresponds to a perfectly mixed system and $U_{s}=1$ corresponds to a perfectly segregated system. In order to remain consistent with mixing efficiency, i.e., higher values corresponding to a better mixed system, the value of $1-U_{s}$ will be used for the comparisons in this work.

The comparison of $1-U_{s}$ for the ramp and strut is shown in Fig $7 \mathrm{p}$. In open-plate mixing experiments such as these, the spatial unmixedness parameter should only be calculated over the intended fueling area for each injector since inclusion of the freestream will bias the value of the parameter (this does not happen for the mixing efficiency due to the integral nature of its calculation). In contrast to the results shown for the mixing efficiency, the results for the spatial unmixedness parameter indicate that the ramp is the better mixer. Because the spatial unmixedness parameter is simply a measure of the spatial variance of the fuel plume's mass fraction across its intended fueling area, what this essentially means is that the ramp does a better job of distributing fuel uniformly across the IFA. However, when mass flux is taken into account by the mixing efficiency, the strut injector is clearly the better mixer. This discrepancy is primarily explained by the the large difference in fuel-to-freestream mass flux ratios between the strut and ramp injectors.

Also contributing to the discrepancy is the natural difference in the mass flux distributions occurring due to the different compressible flow features generated by the differing injector geometries. These differences suggest that if the spatial unmixedness parameter is to be used as the sole metric to determine the performance of multiple injector concepts, then the fuel-to-freestream mass flux ratios of the various injectors should be closer to one another than they are in the current case $\left(\lambda_{\text {strut }} / \lambda_{\text {ramp }} \approx 2.5\right)$ and there should not be substantial geometry or injector configuration differences that cause large natural discrepancies in mass flux across the flowfields of interest.

\section{Decay of Maximum Fuel Mass Fraction}

Tracking the downstream decay of the maximum fuel mass fraction has long been used as a fast and simple metric to compare the relative mixing performance of high-speed fuel injectors [14, 15, 25]. The quantity $1-\alpha_{H e, \text { max }}$ is plotted in Fig $7 \mathrm{~F}$ versus downstream distance for the injectors. Here $1-\alpha_{H e, \max }$ is plotted to again remain consistent with the majority of the metrics of interest where larger values correspond to a better mixed system. Fig 7 ; shows that $\alpha_{H e, \max }$ begins to decay nearer to the injection location for the strut, suggesting, as the mixing efficiency shows, that the strut mixes more rapidly in the near field than the ramp. In the far field, the rate of decay of $\alpha_{H e \text {, max }}$ is greater for the ramp than the strut causing the two curves to intersect at $x=12$ inch. However, caution must be used when interpreting the decay of maximum fuel mass fraction as a global mixing performance metric because it inherently refers to only a single point in the flow at each downstream station.

\section{Plume Area-Based Metrics}

Plume area-based metrics are also commonly used as a substitute metric used to assess fuel/air mixing performance. Plotting the fuel plume area versus downstream distance gives an idea of how much the fuel plume grows by both 
diffusion and entrainment as the flow progresses downstream. Because both the diffusion and entrainment processes aid in mixing the fuel and freestream air, it can generally be said that the more a fuel plume grows, the better the fuel and air are mixed. However, changing the fuel injection pressure and interactions of the plume with shock and expansion waves can alter the plume area without benefiting mixing, so caution is urged when using fuel plume area to evaluate the mixing behavior of an injector concept. Here, the fuel plume area, $A_{p}$, is defined as the area enclosed by the contour of the minimum helium mass fraction that can be measured above the minimum sensitivity of the system by the GSAS, which is $\chi_{H e}=0.01\left(\alpha_{H e} \approx 0.0014\right)$. To take into account different injection total pressure conditions, Fuller et al. [14] defined a relative plume area, $\bar{A}$ as,

$$
\bar{A}=\frac{A_{p}-A_{u}}{A_{s}-A_{u}} .
$$

In Eq.7. $A_{u}$ is the area that a perfectly unmixed jet (i.e., $\alpha_{H e}=1$ ) would expand to if it were allowed to further expand isentropically to the freestream static pressure from its nozzle exit conditions and $A_{s}$ is the area to which a uniformly stoichiometric jet (i.e., $\alpha_{H e}=0.0285$ ) would expand to if also allowed to expand to the same conditions. Therefore, an increase in the total pressure of the fuel injection results in a linear increase of both $A_{u}$ and $A_{s}$. This means that if $\bar{A}$ remains constant when the fuel injection pressure is increased, then the fuel plume is growing proportionally with the injection pressure. Fig $7 \mathrm{~d}$ shows that the relative fuel plume area for the ramp is consistently larger than that of the strut beginning with the first downstream station analyzed, however the rate of plume area growth is very similar for the two configurations.

Another area-based metric is the flammable plume area. The equivalence ratio flammability limits for hydrogen in air are $0.14 \leq \phi \leq 2.54$ [26]. This means that the flammable mass fractions of hydrogen in air lie within the range $0.00397 \leq \alpha_{H_{2}} \leq 0.0675$. Though helium is used here as an inert fuel simulant, the "flammability" limits of helium will be taken to be the same as those of hydrogen. The flammable plume area, $A_{f}$, is defined as the area having a fuel mass fraction within the flammability limits defined above. Both $A_{f}$ and $A_{p}$ are normalized by the IFA and shown in Fig 7 f. Fig 7 shows that the fuel plume of the ramp injector better fills its intended fueling area than the strut injector, consistently filling $10-20 \%$ more of its intended fueling area than the strut. However, whenever the flammable plume area is compared, the strut and ramp injectors fill closer to the same percentage of their intended fueling areas. With both the total fuel plume area and flammable fuel plume area calculated, it is possible to determine the fraction of the fuel plume that is within the defined flammability limits. Fig 7 f depicts the flammable plume fraction for both injector configurations. Of the substitute metrics considered, the flammable plume fraction is the only metric that is generally consistent with the mixing efficiency in indicating that the strut mixes better than the ramp.

\section{E. Correlation of Substitute Metrics with Mixing Efficiency}

Depicted in Fig. 8 are plots of the Richardson extrapolated substitute metrics versus the Richardson extrapolated mixing efficiency for each injector. Also included in this figure are tabulated values of the sample Pearson correlation coefficients between each substitute metric plotted in the figure and the mixing efficiency. Fig. 8 shows the strength of the linear correlation between each substitute metric and the mixing efficiency without considering any changes in the correlations caused by the coarsened resolution and reduced sampling area of the synthetic experimental data.

From Fig. 8, it can be concluded that two metrics, $1-U_{s}$ and $1-\alpha_{H e, \text { max }}$, have appreciably weaker linear correlations with mixing efficiency than the three plume area-based metrics. The correlations between the Richardson extrapolated plume area-based metrics and the mixing efficiency are quite strong, with the sample correlation coefficients surpassing $R=0.97$. Any of the three plume area-based metrics plotted in Fig. 8 could reasonably be used to get a general idea of the shape of the mixing efficiency curve for these two injector configurations. However, with the exception of the flammable plume fraction, the relative performance between the strut and ramp injector, as indicated by these metrics, is opposite to that indicated by the mixing efficiency. Therefore, incorrect conclusions about which injector is the better mixer would be drawn from these metrics even though they are well correlated with the mixing efficiency. Even though the flammable plume fraction is both well correlated with the mixing efficiency and shows the same relative performance between the strut and ramp injector, it cannot definitively be concluded that this metric is, in general, the best substitute metric because only two different injector configurations are compared herein.

\section{Synthetic Experimental Data}

Synthetic experimental data was extracted from the CFD simulations to address two underlying unknowns which will help guide future experimental GSAS test campaigns. Investigated first was the effect that the relatively coarse gas 

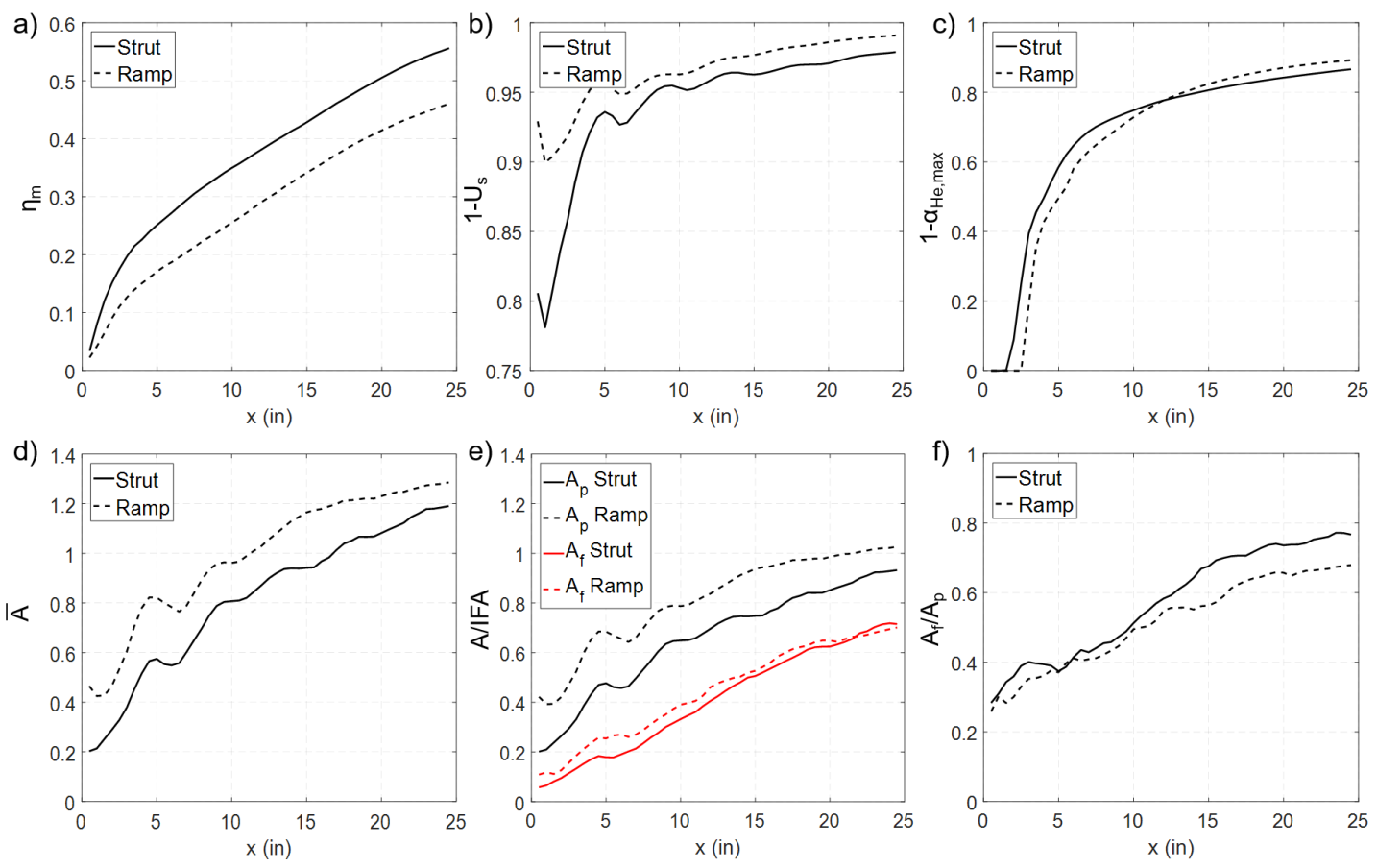

Fig. 7 Richardson extrapolated mixing performance metrics for the strut and ramp injector a) mixing efficiency, b) one minus the spatial unmixedness parameter, c) one minus maximum helium mass fraction, d) relative fuel plume area, e) intended fueling area normalized plume area and flammable plume areas, f) flammable plume fraction.
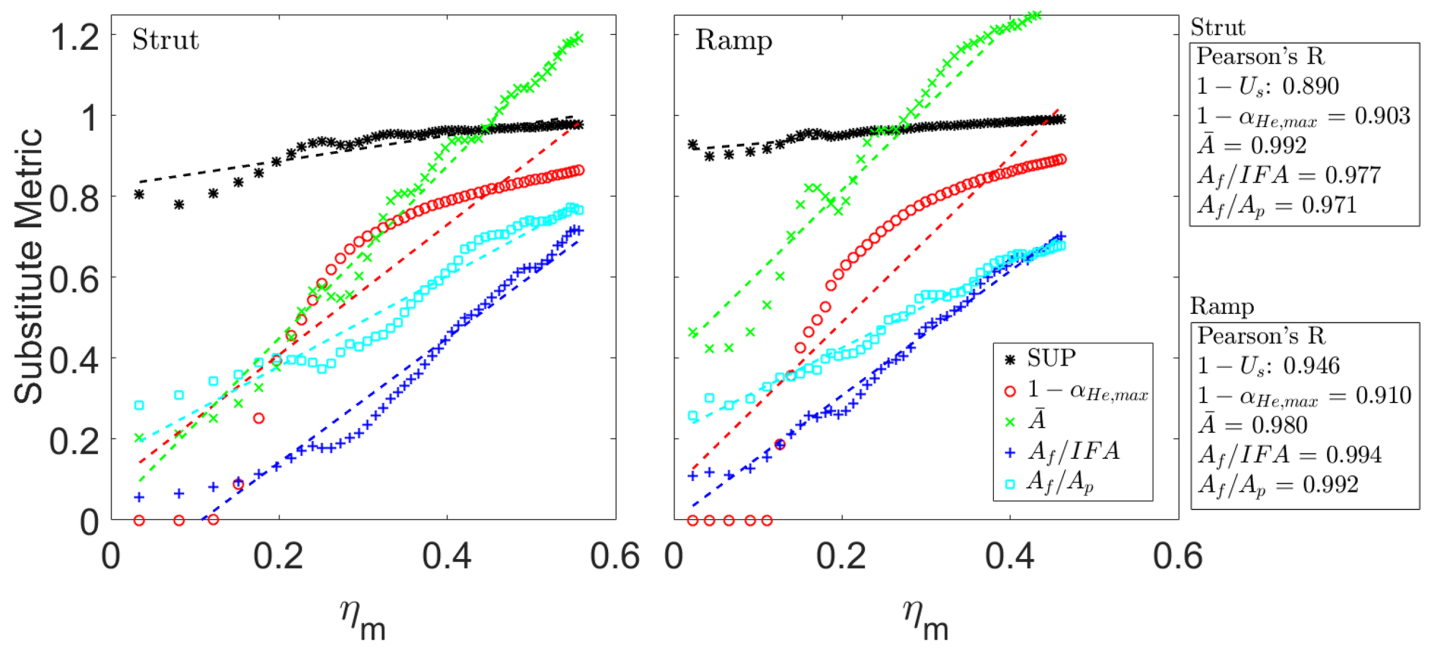

Fig. 8 Richardson extrapolated substitute mixing performance metrics plotted versus $\eta_{m}$ for the strut and ramp injectors. The sample Pearson correlation coefficients of each metric with $\eta_{m}$ are shown at the right of the figure.

sample probe spacing has on the calculation of the substitute mixing performance metrics. This was accomplished by extracting synthetic experimental data at various sample grid resolutions and calculating the root-mean-square 
error (RMSE) between the mixing performance metrics calculated at the sample grid resolution and the Richardson extrapolated value of the same metric. The second, and more important, unknown is the effect that the coarse gas sample grid resolution will have on the correlation between the mixing efficiency and the substitute metrics. This was investigated by calculating the correlation coefficients between the Richardson extrapolated mixing efficiency and the substitute metrics calculated on the coarsened gas sample grid. This will allow for conclusions to be drawn about which substitute metric, sampled at what resolution, is best correlated with the mixing efficiency.

In order to make the synthetic experimental data sets representative of how the actual experimental data will be acquired, the quantities extracted from the CFD data were averaged over the sample probe tip area $(\varnothing=0.035$ inch). Gas sample measurement locations are infinitely adjustable in the $x$ and $z$ directions; however, measurement spacing in the $y$ direction is fixed at either $1 / 4$ inch or $1 / 8$ inch, with the closest measurement to the wall being at a distance of 0.4 inch. Therefore, the calculated RMSE terms include error contributions that arise from both the coarsened sample grid spacing and the fact that fuel plume measurements begin 0.4 inch from the flat plate. In order to highlight how the coarsened grid resolution affects the synthetic experimental data sets, Fig. 9 depicts the helium mass fraction contours for the strut injector at the $x=1.0$ inch downstream station for various sample grid resolutions.

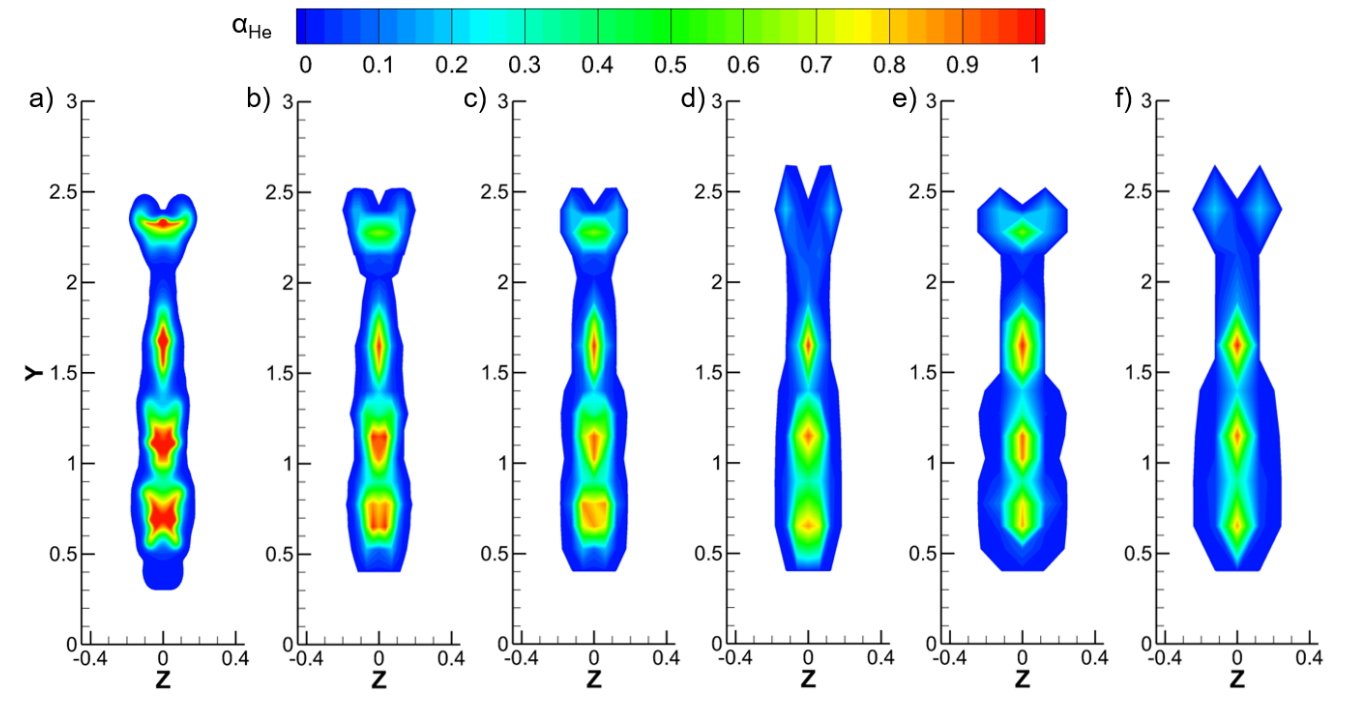

Fig. 9 Helium mass fraction contours at $x=1.0$ inch for strut injector at a) fine CFD resolution b) $0.035 \times 1 / 8$ in. $(z \times y$ probe spacing), c) $1 / 16 \times 1 / 8$ in., d) $1 / 16 \times 1 / 4$ in., e) $1 / 8 \times 1 / 8$ in., f) $1 / 8 \times 1 / 4$ in.

The root-mean-square errors for each metric at various sample grid resolutions are tabulated for comparison in Table 2 Because the possible ranges of each substitute metric are all approximately from zero to one, the magnitude of the RMSEs can be used to directly assess which metrics are most accurately calculated over the coarsened grids of the synthetic experimental data. Depicted in Fig. 10 are the substitute mixing performance metrics calculated with the synthetic experimental data plotted versus the Richardson extrapolated mixing efficiency. The resolution of the synthetic experimental data plotted in Fig. 10 corresponds to that with the lowest RMSE for that particular metric for each injector configuration.

The data presented in Table 2 and Fig. 10 allow for multiple conclusions to be drawn about the ability of the various substitute mixing performance metrics to serve as good indicators of mixing efficiency. As indicated by the data in Table 2, the substitute metric which is most accurately calculated from the synthetic experimental data sets is the spatial unmixedness parameter, $1-U_{s}$. However, this metric is poorly correlated with mixing efficiency when compared to the plume area-based metrics. The same can be said for the decay of maximum fuel mass fraction, which is calculated more accurately from the synthetic experimental data sets than the plume area-based metrics but clearly does not have a linear relationship with mixing efficiency. Table 2 shows that the plume area-based metrics have the largest RMSEs between their Richardson extrapolated values and their values when calculated with the synthetic experimental data. This is to be expected and can be inferred from Fig. 9 since the various plume areas are calculated simply by summing the areas of the individual grid cells that have a helium mass fraction value above a certain value. Interestingly, Table 2 
Table 2 Root-mean-square error between Richardson extrapolated and synthetic experimental mixing performance metrics. Minimum RMSE for each metric is in bold for each injector configuration

\begin{tabular}{lc|cccccc}
\hline \hline \multicolumn{2}{l}{ Simulated Experiment Resolution } & $\mathbf{1}-\boldsymbol{U}_{\boldsymbol{S}}$ & $\boldsymbol{\alpha}_{\boldsymbol{H} \boldsymbol{e}, \boldsymbol{m a x}}$ & $\overline{\boldsymbol{A}}$ & $\boldsymbol{A}_{\boldsymbol{p}} / \boldsymbol{I F} \boldsymbol{A}$ & $\boldsymbol{A}_{\boldsymbol{f}} / \boldsymbol{I F} \boldsymbol{A}$ & $\boldsymbol{A}_{\boldsymbol{f}} / \boldsymbol{A}$ \\
\hline \multirow{2}{*}{$1 / 8 \times 1 / 4$ in. } & Strut & 0.0097 & 0.0493 & 0.1079 & 0.0798 & 0.1903 & 0.0797 \\
& Ramp & 0.0129 & 0.0454 & 0.2432 & 0.1791 & 0.2182 & 0.0909 \\
\hline \multirow{2}{*}{$1 / 16 \times 1 / 4$ in. } & Strut & $\mathbf{0 . 0 0 7 5}$ & 0.0493 & $\mathbf{0 . 0 8 8 2}$ & $\mathbf{0 . 0 5 7 0}$ & 0.2088 & 0.0521 \\
& Ramp & $\mathbf{0 . 0 1 0 7}$ & 0.0444 & $\mathbf{0 . 2 1 2 6}$ & $\mathbf{0 . 1 5 6 6}$ & 0.2332 & $\mathbf{0 . 0 8 1 2}$ \\
\hline \multirow{2}{*}{$1 / 8 \times 1 / 8$ in. } & Strut & 0.0101 & $\mathbf{0 . 0 1 6 5}$ & 0.1225 & 0.0906 & $\mathbf{0 . 1 7 8 8}$ & 0.0720 \\
& Ramp & 0.0157 & 0.0392 & 0.2950 & 0.2172 & $\mathbf{0 . 1 8 7 2}$ & 0.1449 \\
\hline \multirow{2}{*}{$1 / 16 \times 1 / 8$ in. } & Strut & 0.0084 & 0.0165 & 0.1085 & 0.0803 & 0.1833 & $\mathbf{0 . 0 4 5 4}$ \\
& Ramp & 0.0137 & 0.0385 & 0.2666 & 0.1963 & 0.1956 & 0.1269 \\
\hline \multirow{2}{*}{$0.035 \times 1 / 8$ in. } & Strut & 0.0137 & 0.0165 & 0.1116 & 0.0825 & 0.1856 & 0.0538 \\
& Ramp & 0.0125 & $\mathbf{0 . 0 2 8 5}$ & 0.2517 & 0.1854 & 0.2025 & 0.1166 \\
\hline \hline
\end{tabular}
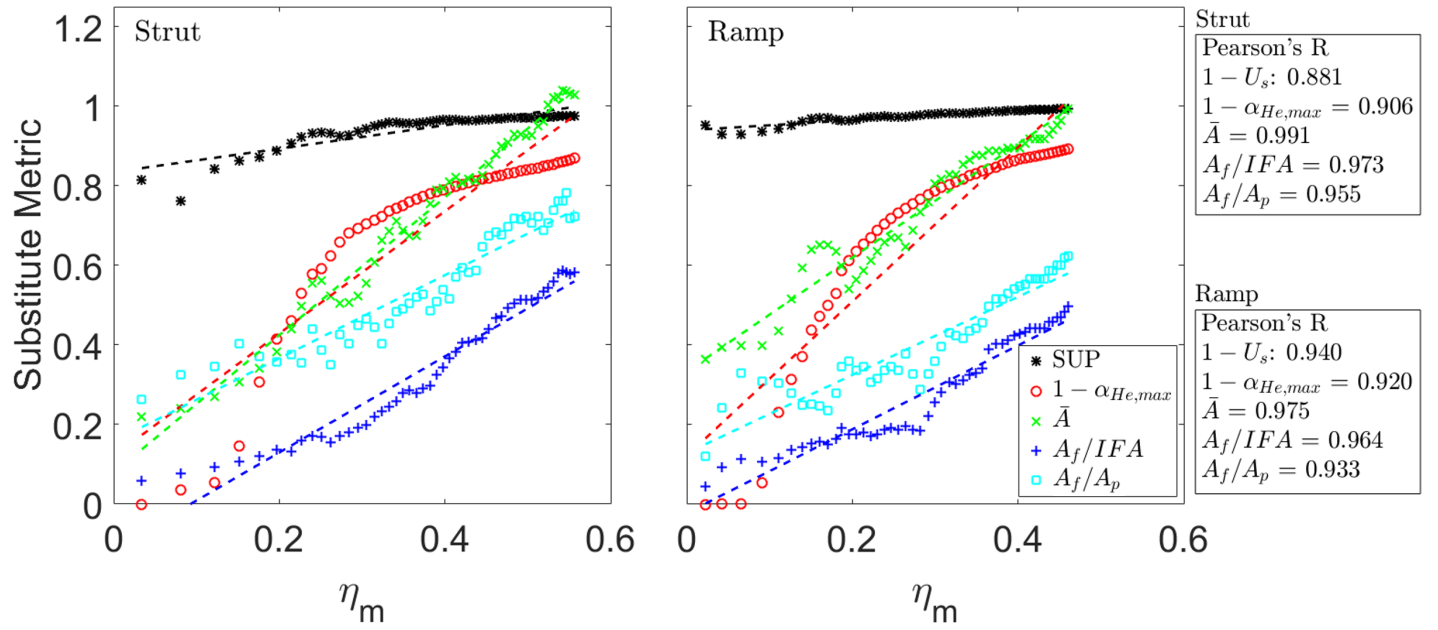

Fig. 10 Substitute mixing performance metrics calculated with synthetic experimental data plotted versus $\eta_{m}$ for the strut and ramp injector configurations. The sample Pearson correlation coefficients of each metric with $\eta_{m}$ are shown at the right of the figure.

shows that the finest resolution of the synthetic experimental data sets is not the most accurate at reproducing the Richardson extrapolated plume area-based metrics. However, this occurrence should not be used to draw the conclusion that any specific coarser resolution is necessarily better than the finest resolution because the area-based metrics are also dependent on the individual probe locations within the plume so a shift of the entire grid in relation to the fuel plume can substantially change the values of these metrics. This fact makes choosing an experimental resolution with which to sample the flowfields to get the most accurate values of plume area-based mixing metrics a challenging task without knowing the fuel distribution beforehand. The last major fact from Table 2 that should be highlighted is that the RMSEs of the plume area-based metrics for the ramp injector are consistently higher than those for the strut. This is due to the fact that a larger fraction of the fuel plume is not recovered for the ramp injector because more of its fuel plume is closer to the wall and falls below the first vertical sample point $(y=0.4 \mathrm{inch})$ in the grid of the synthetic experimental data. Comparing Fig. 8 to Fig. 10 it is evident that the strength of the correlations of the substitute metrics to the mixing efficiency invariably decrease a few percent when the synthetic experimental data is used, however their relationships to one another generally remain the same. 
The primary outcome that should be highlighted from the results of this analysis is that when comparing the fuel/air mixing performance of high-speed fuel injectors having significant differences in geometrical configuration and/or fuel injection parameters, conclusive comparisons about which injector mixes better generally should be made with a mass flux-weighted mixing performance metric. However, this is not to say that the various one-dimensional substitute mixing performance metrics are not without value-quite the opposite is true. For one, when these metrics are experimentally measured, they can be used to anchor and compare to the metrics calculated from CFD simulations. Secondly, previous results in the literature have shown that for injectors of similar geometry and injection conditions (where the mass flux distribution will, naturally, be more similar), these substitute metrics generally yield the same hierarchy of relative performance as the mixing efficiency [14, 15], suggesting that these substitute metrics could be used to identify the best mixer out of a family of similar injector configurations if the measurements have low enough uncertainty. In the future, as the EIMP computational and experimental database of injector configurations increases, the substitute mixing performance metrics will be cataloged in order to gain a better perspective on their behavior and relationship to the mixing efficiency for a wider variety of high-speed injector configurations.

\section{Summary and Conclusions}

The work presented herein has compared several different one-dimensional fuel/air mixing performance metrics for two different high-speed fuel injector configurations, a strut and a ramp. These types of injector configurations are both commonly used to distribute the fuel in scramjet combustors. Specifically, this work has assessed the correlation of several fuel distribution-based metrics with the mass flux-weighted mixing efficiency. This was done because, even though the mixing efficiency is the metric that is best suited to compare the overall mixing performance of a batch of injectors, its calculation requires the measurement of the gas composition and at least three independent aerothermodynamic variables - thereby complicating its experimental determination. For this reason, several substitute metrics, which are more readily determined experimentally, are commonly used to assess mixing performance in lieu of the mixing efficiency. However, because these substitute metrics do not provide a mass flux-weighted measure of mixing completeness, it is important to understand their relationship to the mixing efficiency. The substitute mixing performance metrics investigated were the spatial unmixedness parameter, decay of maximum fuel mass fraction, intended fueling area normalized fuel plume and flammable plume areas, relative fuel plume area, and flammable plume fraction. These metrics are able to be measured with the current experimental setup of the Enhanced Injection and Mixing Project at the NASA Langley Research Center, which includes in-stream gas sampling, Pitot pressure, and total temperature measurements. Using previously generated CFD data, synthetic experimental data sets were generated by extracting data points from the CFD simulations at the locations that are intended to be probed experimentally.

Comparing the substitute metrics calculated from the synthetic experimental data sets revealed that the spatial unmixedness parameter and the decay of fuel mass fraction are most accurately calculated over the coarsened grids, yet have a lower correlation with the mixing efficiency than do the plume area-based metrics. The plume area-based metrics, which have a strong correlation with the mixing efficiency, do not consistently yield the same relative performance between the ramp and the strut as the mixing efficiency does, meaning that incorrect conclusions about which injector is the better mixer can be drawn from these metrics when used in place of the mixing efficiency. Therefore, it is recommended that when comparing multiple injectors with significantly different geometries or injection configurations, as is done in this work, that these substitute mixing performance metrics be used primarily to anchor comparisons with the results from CFD simulations and not to draw definitive conclusions about which injector better mixes fuel and air.

\section{References}

[1] McClinton, C. R., "X-43-Scramjet Power Breaks the Hypersonic Barrier Dryden Lectureship in Research for 2006," 44th AIAA Aerospace Sciences Meeting and Exhibit, AIAA 2006-1, Reno, NV, 2006.

[2] Hank, J. M., Murphy, J. S., and Mutzman, R. C., “The X-51A Scramjet Engine Flight Demonstration Program,” 15th AIAA International Space Planes and Hypersonic Systems and Technologies Conference, AIAA 2008-2540, Dayton, OH, 2008.

[3] Kutschenreuter, P., "Supersonic Flow Combustors," Scramjet Propulsion, edited by E. T. Curran and S. N. B. Murthy, Progress in Astronautics and Aeronautics, AIAA, Reston, VA, 2000, pp. 513-568.

[4] Seiner, J. M., Dash, S. M., and Kenzakowski, D. C., "Historical Survey on Enhanced Mixing in Scramjet Engines," 9th International Space Planes and Hypersonic Systems and Technologies Conference, AIAA 99-4869, Norfolk, VA, 1999. 
[5] Gutmark, E. J., Schadow, K. C., and Yu, K. H., "Mixing Enhancement in Supersonic Free Shear Flows," Annual Review of Fluid Mechanics, Vol. 27, 1995, pp. 375-417. doi:10.1146/annurev.fl.27.010195.002111.

[6] Lee, J., Lin, K., and Eklund, D., "Challenges in Fuel Injection for High-Speed Propulsion Systems," AIAA Journal, Vol. 53, No. 6, 2015, pp. 1405-1423. doi:10.2514/1.J053280.

[7] Cabell, K., Drozda, T. G., Axdahl, E. L., and Danehy, P. M., "The Enhanced Injection and Mixing Project at NASA Langley,” JANNAF 46th CS / 34th APS / 34th EPSS / 28th PSHS Joint Subcommittee Meeting, Albuquerque, NM, 2014.

[8] Drozda, T. G., Cabell, K. F., Ziltz, A. R., Hass, N. E., Inman, J. A., Burns, R. A., Bathel, B. F., Danehy, P. M., Abdul-Huda, Y. M., and Gamba, M., "Comparisons Between NO PLIF Imaging and CFD Simulations of Mixing Flowfields for High-Speed Fuel Injectors," 53rd AIAA/SAE/ASEE Joint Propulsion Conference, AIAA Propulsion and Energy Forum, AIAA 2017-4647, Atlanta, GA, 2017.

[9] Drozda, T. G., Cabell, K. F., Ziltz, A. R., Hass, N. E., Inman, J. A., Burns, R. A., Bathel, B. F., and Danehy, P. M., "Comparisons Between NO PLIF Imaging and CFD Simulations of Mixing Flowfields for High-Speed Fuel Injectors," JANNAF 48th CS / 36th APS / 36th EPSS / 30th PSHS / PIB Joint Subcommittee Meeting, Newport News, VA, 2017.

[10] Drozda, T. G., Drummond, J. P., and Baurle, R. A., "CFD Analysis of Mixing Characteristics of Several Fuel Injectors at Hypervelocity Flow Conditions," 52nd AIAA/SAE/ASEE Joint Propulsion Conference, AIAA Propulsion and Energy Forum, AIAA 2016-4764, Salt Lake City, UT, 2016.

[11] Drozda, T. G., Baurle, R. A., and Drummond, J. P., "Impact of Flight Enthalpy, Fuel Simulant, and Chemical Reactions on the Mixing Characteristics of Several Injectors at Hypervelocity Flow Conditions," 63rd JANNAF Propulsion Meeting / JANNAF 47th CS / 35th APS / 35th EPSS / 29th PSHS / Joint Subcommittee Meeting, Newport News, VA, 2016.

[12] Drozda, T. G., Shenoy, R. R., Passe, B. J., Baurle, R. A., and Drummond, J. P., "Comparison of Mixing Characteristics for Several Fuel Injectors on an Open Plate and in a Ducted Flowpath Configuration at Hypervelocity Flow Conditions," JANNAF 48th CS / 36th APS / 36th EPSS / 30th PSHS / PIB Joint Subcommittee Meeting, Newport News, VA, 2017.

[13] Liscinsky, D. S., True, B., and Holdeman, J. D., "Experimental Investigation of Crossflow Jet Mixing in a Rectangular Duct," 29th AIAA/SAE/ASME/ASEE Joint Propulsion Conference and Exhibit, AIAA 93-2037, Monterey, CA, 1993.

[14] Fuller, R. P., Wu, P., Nejad, A. S., and Schetz, J. A., "Comparison of Physical and Aerodynamic Ramps as Fuel Injectors in Supersonic Flow," Journal of Propulsion and Power, Vol. 14, No. 2, 1998, pp. 135-145. doi:10.2514/2.5278.

[15] Doster, J. C., King, P. I., Gruber, M. R., Carter, C. D., Ryan, M. D., and Hsu, K. Y., "In-Stream Hypermixer Fueling Pylons in Supersonic Flow,” Journal of Propulsion and Power, Vol. 25, No. 4, 2009, pp. 885-901. doi:10.2514/1.40179.

[16] Baurle, R. A., Fuller, R. P., White, J. A., Chen, T. H., Gruber, M. R., and Nejad, A. S., “An Investigation of Advanced Fuel Injection Schemes for Scramjet Combustion,” 36th AIAA Aerospace Sciences Meeting and Exhibit, 1998.

[17] VULCAN-CFD, “http://vulcan-cfd.larc.nasa.gov/,”, Dec. 2017.

[18] van Leer, B., “Towards the Ultimate Conservative Difference Scheme. V: A Second-Order Sequel to Godunov's Method," Journal of Computational Physics, Vol. 32, No. 1, 1979, pp. 101-136. doi:10.1016/0021-9991(79)90145-1.

[19] Edwards, J. R., “A Low-Diffusion Flux-Splitting Scheme for Navier-Stokes Calculations," Computers and Fluids, Vol. 26, No. 6, 1997, pp. 635-659. doi:10.1016/S0045-7930(97)00014-5.

[20] McBride, B. J., Gordon, S., and Reno, M. A., "Thermodynamic Data for Fifty Reference Elements," NASA Technical Paper 3287/REV1, NASA, Cleveland, OH, Feb. 2001.

[21] Pulliam, T. H., and Chaussee, D. S., "A Diagonal Form of an Implicit Approximate-Factorization Algorithm," Journal of Computational Physics, Vol. 39, No. 2, 1981, pp. 347-363. doi:10.1016/0021-9991(81)90156-X.

[22] Menter, F. R., “Two-Equation Eddy-Viscosity Turbulence Models for Engineering Applications," AIAA Journal, Vol. 32, No. 8, 1994, pp. 1598-1605. doi:10.2514/3.12149.

[23] Wilcox, D. C., Turbulence Modeling for CFD, DCW Industries, Inc., La Cañada, CA, 2000.

[24] Roache, P. J., Verification and Validation in Computational Science and Engineering, Hermosa Publishers, 1998.

[25] Mao, M., Riggins, D. W., and McClinton, C. R., "Numerical Simulation of Transverse Fuel Injection," Computational Fluid Dynamics Symposium on Aeropropulsion, NASA, Cleveland, OH, 1990, pp. 635-667.

[26] Glassman, I., and Yetter, R. A., Combustion, $4^{\text {th }}$ ed., Academic Press, Inc., San Diego, CA, 2008. 